\title{
An FPGA-based bolometer for the MAST-U Super-X divertor
}

Cite as: Rev. Sci. Instrum. 87, 11 721 (2016); https://doi.org/10.1063/1.4961556

Submitted: 08 June 2016 . Accepted: 27 July 2016 . Published Online: 11 November 2016

Jack Lovell, Graham Naylor, Anthony Field, Peter Drewelow, Ray Sharples (D), and JET Contributors

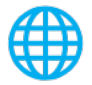

\section{ARTICLES YOU MAY BE INTERESTED IN}

A low noise highly integrated bolometer array for absolute measurement of VUV and soft $\mathrm{x}$ radiation

Review of Scientific Instruments 62, 744 (1991); https://doi.org/10.1063/1.1142078

Modifications to the synthetic aperture microwave imaging diagnostic

Review of Scientific Instruments 87, 11E129 (2016); https://doi.org/10.1063/1.4961283

Controlling femtosecond-laser-driven shock-waves in hot, dense plasma

Physics of Plasmas 24, 072702 (2017); https://doi.org/10.1063/1.4990059

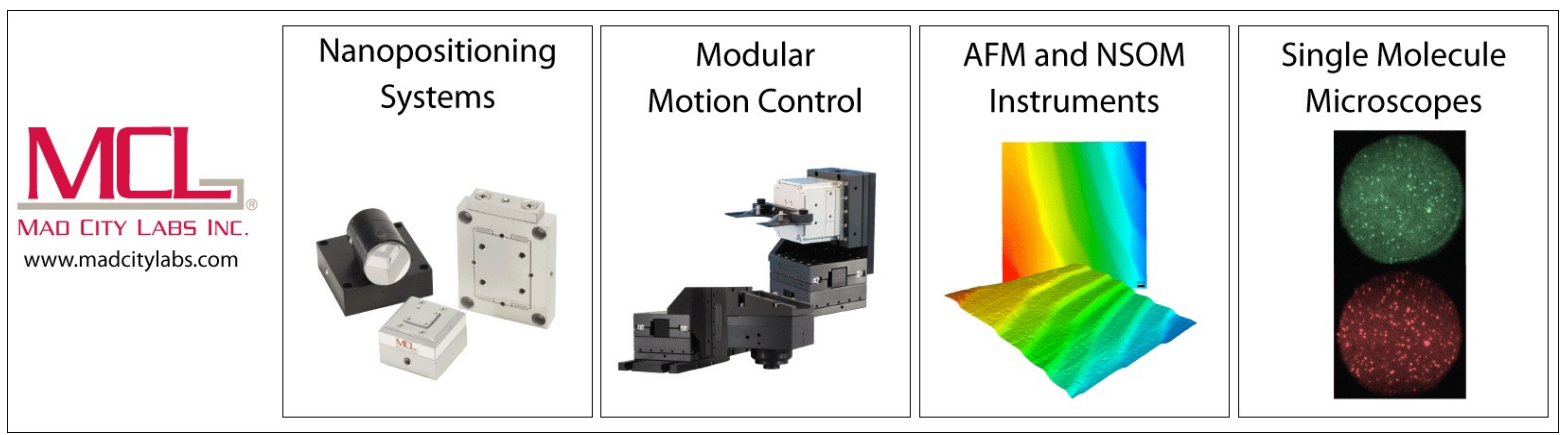




\title{
An FPGA-based bolometer for the MAST-U Super-X divertor
}

\author{
Jack Lovell, ${ }^{1,2, a)}$ Graham Naylor, ${ }^{2}$ Anthony Field, ${ }^{2}$ Peter Drewelow, ${ }^{3}$ Ray Sharples, ${ }^{1}$ \\ and JET Contributors $\left.{ }^{4, b}\right)$ \\ ${ }^{1}$ Durham University, South Road, Durham DH1 3LE, United Kingdom \\ ${ }^{2}$ Culham Centre for Fusion Energy, Culham Science Centre, Abingdon, Oxon OX14 3DB, United Kingdom \\ ${ }^{3}$ MPI für Plasmaphysik, Greifswald, Germany \\ ${ }^{4}$ EUROfusion Consortium, JET, Culham Science Centre, Abingdon OX14 3DB, United Kingdom
}

(Presented 9 June 2016; received 8 June 2016; accepted 27 July 2016;

published online 29 August 2016)

A new resistive bolometer system has been developed for MAST-Upgrade. It will measure radiated power in the new Super-X divertor, with millisecond time resolution, along 16 vertical and 16 horizontal lines of sight. The system uses a Xilinx Zynq-7000 series Field-Programmable Gate Array (FPGA) in the D-TACQ ACQ2106 carrier to perform real time data acquisition and signal processing. The FPGA enables AC-synchronous detection using high performance digital filtering to achieve a high signal-to-noise ratio and will be able to output processed data in real time with millisecond latency. The system has been installed on 8 previously unused channels of the JET vertical bolometer system. Initial results suggest good agreement with data from existing vertical channels but with higher bandwidth and signal-to-noise ratio. [http://dx.doi.org/10.1063/1.4961556]

\section{INTRODUCTION}

Bolometers are widely used for radiation measurements in fusion devices. Bolometer measurements are important for power balance studies, and arrays of detectors can be used to make spatially resolved measurements of plasma radiation, enabling an improved understanding of radiative losses.

The MAST spherical tokamak at Culham Centre for Fusion Energy (CCFE) is currently undergoing a major upgrade. A significant feature of the upgraded device (MAST$\mathrm{U})$ is the new "Super-X" divertor (SXD) configuration, which aims to reduce heat load on the divertor target plates. ${ }^{1}$ However, this is one of the first fusion devices to use this particular divertor configuration, so a thorough and highquality diagnosis of the SXD is imperative. The large number of divertor magnetic field coils, and gas injection valves, in the MAST-U tokamak allow the possibility of real-time control applications to optimize divertor operation. It is therefore advantageous to have diagnostics which cannot only make high quality measurements, but also produce low-latency real time data to be used in control and feedback systems.

The new bolometer system which has been developed for MAST-U has already been introduced in a previous paper. ${ }^{2}$ In this paper, we describe in more detail the capabilities of the system. We then go on to demonstrate operation of the system on the JET tokamak and compare the results obtained with those of the existing JET bolometer system.

Note: Contributed paper, published as part of the Proceedings of the 21st Topical Conference on High-Temperature Plasma Diagnostics, Madison, Wisconsin, USA, June 2016.

a)jack.lovell@durham.ac.uk

b) See the Appendix of F. Romanelli et al., Proceedings of the 25th IAEA Fusion Energy Conference 2014, Saint Petersburg, Russia.

\section{CAPABILITIES OF THE NEW SYSTEM}

The system as developed for MAST-U comprises 32 bolometer sensors with lines of sight in the divertor. These sensors are of the same Pt/SiN design as is to be used in ITER. ${ }^{3}$ 16 sensors view the chamber vertically and 16 horizontally. Figure 1 shows these lines of sight, with a simulated emissivity profile as calculated from the SOLPS code. ${ }^{4}$ The configuration allows us to reconstruct the $2 \mathrm{D}$ emissivity profile from integral line of sight measurements using tomography.

The new system uses Field-Programmable Gate Array (FPGA) technology. A Xilinx Zynq System-On-Chip (SOC), combining a dual core ARM CPU running Linux and FPGA programmable logic, is used to control the excitation of the 32 bolometer sensors, digitisation of the sensor output voltages, and processing of the signals. The electronics hardware is built by D-TACQ Solutions, ${ }^{5}$ the digital signal processing to be performed on the FPGA is designed by Durham University and CCFE. A digital acquisition system was chosen instead of an analogue one as it offers improved signal quality, greater compactness, and a significant reduction in cost per channel.

In common with previous resistive bolometer systems, which consist of a Wheatstone bridge with two resistors heated by plasma radiation and two resistors shielded, the detector signals are measured using $\mathrm{AC}$ synchronous detection. ${ }^{6} \mathrm{An} \mathrm{AC}$ excitation voltage in the form of a sine wave, at a frequency of about $20 \mathrm{kHz}$, is applied across one diagonal of the bridge, and from the output voltage across the other diagonal, we measure the amplitude only at the excitation frequency. This technique allows us to extract the small bolometer signal from the noisy environment of a tokamak. It involves mixing the bolometer output signal with a reference signal of the correct frequency and phase, and low-pass filtering the mixed signal to remove the mixing harmonics and out-of-band noise. Traditionally, this has been performed using analogue electronics, which are 


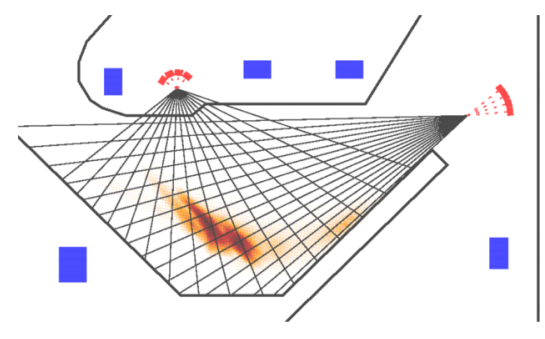

FIG. 1. Illustration of the lines of sight of the SXD bolometer system on MAST-U.

susceptible to deterioration over time and to manufacturing tolerances.

In the new system, this processing is performed digitally. The bridge voltage is digitised, then multiplied by in-phase and quadrature-phase copies of the excitation voltage, and the results are filtered using digital finite impulse response (FIR) filters. This produces I and Q components, which can be considered as real and imaginary parts of the signal, and so can be converted to polar representation to extract the voltage amplitude. This process has the additional benefit of being phase sensitive: the true amplitude is produced whatever the phase of the bridge signal relative to the reference signal, meaning that it is not necessary to manually compensate for any phase delays in the system.

The excitation frequency is configurable. This allows the system to be run at a frequency away from other sources of noise on the tokamak, such as switching power supplies. Additionally, the filter bandwidth is configurable up to $2 \mathrm{kHz}$. Higher bandwidth is suitable for measuring large transient events, whereas lower bandwidth filters will allow measurement of very small but slowly evolving signals.

Once the amplitude $A$ has been measured, the power incident on the bolometer sensor can be calculated,

$$
P=\frac{1}{S}\left(A+\tau \frac{\mathrm{d} A}{\mathrm{~d} t}\right) .
$$

This is a simplified form of the more complete expression derived by Giannone et l. $^{7}$ This form has the advantage of only depending on the measured voltage amplitude and two calibration constants: the sensitivity $S(\mathrm{~V} / \mathrm{W})$ and cooling time constant $\tau$. The calibration procedure is described in detail in Section 5 of Ref. 2. It can be performed for every sensor simultaneously, is fully automated, and takes only a few seconds, meaning that it can be performed before every plasma shot.

The use of digital FIR filters allows for more complex signal processing than analogue filters. Using knowledge of the sensitivity and cooling time constants, it is possible to design a filter kernel that will simultaneously differentiate the voltage signals, multiply by the cooling time, and add this time-derivative to the original signals, in addition to low-pass filtering the signals. This means we can actually calculate $P$ for each sensor using Equation (1) on the FPGA in real time, and these data are available to send over a fibre-optic network to a control system for use in a feedback loop. The signal latency is determined by the time taken for samples to pass through the filter system and is of order $1 \mathrm{~ms}$.
By combining the real-time calculated power values described in the previous paragraph with knowledge of the geometry of the sensors, it is possible to calculate moments of the emissivity profile, without having to do a full tomographic inversion. The 1 st and 2 nd order moments give the average position and the size of the emission, respectively. This is particularly useful for the MAST-U divertor, since optimising the size and location of the emitting region will cool the plasma exhaust and help to reduce the heat load on the divertor target. Some work on an FPGA implementation of this processing has already been done, ${ }^{8}$ though it has yet to be integrated into the bolometer system. By performing this processing on the FPGA, we can reduce the workload of the plasma control system and deliver the calculation results with a known fixed latency.

\section{INSTALLATION ON JET}

The new electronics has been installed on the JET tokamak, using 8 previously unused channels of the existing vertical bolometer system. The lines of sight are shown in Figure 2. Although represented by lines, each channel actually views a finite solid angle, extending half-way to the neighbouring channel, giving complete coverage of the plasma. The same is true of the channels on the existing JET system, though there are more of these (24 vertical and 28 horizontal) with closer spacing and hence narrower viewing angles. Some lines of sight are shared between the existing system and the new system, meaning that it is possible to directly compare measurements from the two systems, as long as the different viewing geometries are taken into account.

Figure 3 shows a comparison between two channels with similar lines of sight. The new system does not have a high gain amplifier like the old system, so the voltage measurements are much smaller. However, it can clearly be seen that the two systems are in qualitative agreement. Furthermore, there is a higher signal-to-noise ratio (SNR), despite the filters in the new system being set to $1 \mathrm{kHz}$ bandwidth in this pulse, compared to $200 \mathrm{~Hz}$ for the existing system. This demonstrates that we can make higher bandwidth measurements without compromising on signal quality. When differentiating this signal to calculate the incident power using Equation (1), high

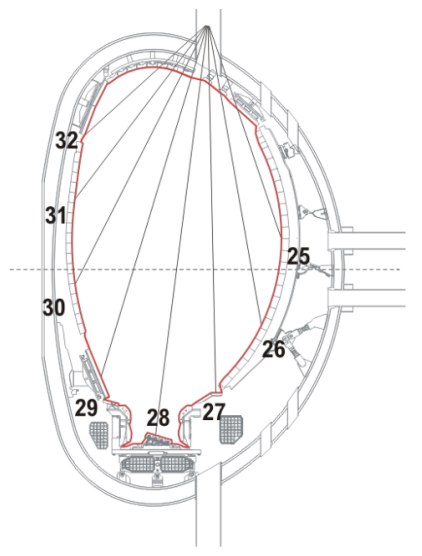

FIG. 2. Lines of sight of bolometer system, installed on JET. 


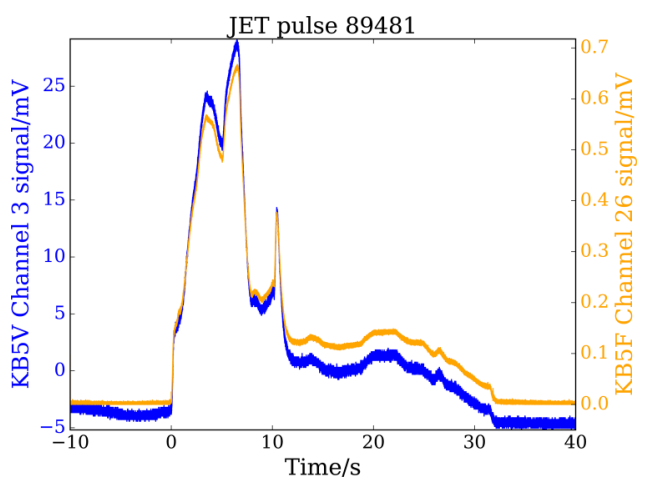

FIG. 3. Comparison of channel 3 of the existing JET system (KB5V, dark blue) and channel 26 of the new system (KB5F, light orange). Note that the zero point on the KB5F axis has been shifted to avoid the two traces overlapping too closely.

frequency noise is amplified. The new system will therefore have an even higher SNR when calculating the power for the same bandwidth.

To perform a quantitative validation of the new system's data, the measured voltage was used to calculate the lineintegrated intensity for each channel, $I=4 \pi P / E$, where $P$ is calculated using Equation (1) and $E$ is the étendue of the sensor. The expected intensity measurement can be obtained by integrating a tomographic reconstruction of the emissivity profile along the channel's line of sight. The tomographic reconstruction was performed using only data from the existing JET system, and the back-calculation was done additionally for the new system. Figure 4 shows the result of this for JET pulse 89548 at one of the time slices for

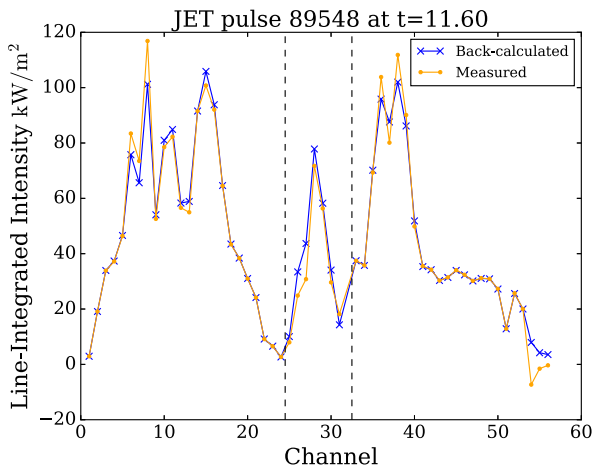

FIG. 4. Comparison of measured intensity and expected intensity, backcalculated from the emissivity profile. The channels belonging to the new system (KB5F) are inside the dashed lines. The vertical channels of the existing system are on the left of the plot and the horizontal channels on the right. which a valid reconstruction was available. Channel 32 has been omitted, since it was discovered upon installation of the electronics that the in-vessel sensor for this channel is broken. The measured and expected intensities are in good agreement, with deviations in the new system comparable to those of the existing system. The agreement of the new system with calculations using the existing system's data demonstrates that the new system has accuracy comparable to that of the existing system.

\section{SUMMARY AND CONCLUSIONS}

The new bolometer system which has been developed for the MAST-U Super-X divertor has been described. Improvements over existing bolometer systems include the use of an FPGA to perform digital signal processing, a fast and automated calibration procedure, and the ability to calculate the power incident on the bolometer sensors in real time. Future real time control applications have been discussed. The electronics have been installed on the JET tokamak on 8 previously unused vertical channels, and the system has been shown to produce data in good agreement with the existing JET bolometer system, but with a better SNR, even at higher bandwidth.

\section{ACKNOWLEDGMENTS}

This work was supported by the Engineering and Physical Sciences Research Council (Grant NO. EP/L01663X/1). This work has been carried out within the framework of the EUROfusion Consortium and has received funding from the Euratom research and training programme 2014-2018 under Grant Agreement No. 633053. The views and opinions expressed herein do not necessarily reflect those of the European Commission.

${ }^{1}$ P. M. Valanju, M. Kotschenreuther, S. M. Mahajan, and J. Canik, Phys. Plasmas 16, 056110 (2009).

${ }^{2}$ J. Lovell, G. Naylor, A. Field, and R. Sharples, in Proceedings of the 1st EPS Conference on Plasma Diagnostics (POS, 2015).

${ }^{3}$ L. Giannone, D. Queen, F. Hellman, and J. C. Fuchs, Plasma Phys. Controlled Fusion 47, 2123 (2005).

${ }^{4}$ R. Schneider, X. Bonnin, K. Borrass, D. P. Coster, H. Kastelewicz, D. Reiter, V. A. Rozhansky, and B. J. Braams, Contrib. Plasma Phys. 46, 3 (2006).

${ }^{5}$ See http://www.d-tacq.com for contact details.

${ }^{6}$ K. F. Mast, J. C. Vallet, C. Andelfinger, P. Betzler, H. Kraus, and G. Schramm, Rev. Sci. Instrum. 62, 744 (1991).

${ }^{7}$ L. Giannone, K. Mast, and M. Schubert, Rev. Sci. Instrum. 73, 3205 (2002). ${ }^{8}$ J. Harrison, "Reconstruction of real time plasma emission information from line integrals using an FPGA," Master's thesis, University of Oxford, 2012. 